\title{
A Graphic Modeling Method of Thermal Hydraulic System in Power Plant Based on MUSE
}

\author{
Yaqing $\mathrm{ZHU}^{1}$, Fengping $\mathrm{PAN}^{2}$, Lingling $\mathrm{SHI}^{3}$, Zhiqiang $\mathrm{PANG}^{4}$, Juanjuan \\ $\mathrm{REN}^{5}$, Shengfang $\mathrm{YU}^{6, a}$ \\ 1,2,3,4,5Electric Power Research Institute of Guangdong Grid Company, Guangzhou 510080, China \\ ${ }^{6}$ UNI Group (Beijing) Intelligent Control Science \& Technology Co., Ltd., Beijing 100085, China \\ aemail: yushengfang@unicst.com
}

Keywords: thermal hydraulic; graphic modeling; module; simulation

\begin{abstract}
Based on the MUSE simulation support environment, designed a complete graphic modeling equation of thermal hydraulic system. Developed a module library, source files library, and a series of manage and configuration tools software. This software package is complete opened and orient. It has a simple instantiation, debug, and automatic create document method. The application in the practice is well.
\end{abstract}

\section{Introduction}

With the development of the power plant simulation technology, the research and development process of large power plant simulation training devices have become increasingly demanding Due to thermal and fluid network system will always appear at the same time, the coupling relationship is strong, the fluid network shows strong nonlinear, so also more complicated to handle[1]. Therefore this paper introduced a new distributed object-oriented simulation supporting environment MUSE (Multi-User Simulation Environment), developed a set of the power plant system graphic modeling tool software, which is designed with the method below: it takes actually existing physics object as independent module, to build its mathematical model, and through analyzing the connection between modules, the system automatically recognizes network topological structure hidden in the system flow process. By using the unique fluid network algorithm of our company, it can finish the calculation of the large-scale fluid network automatically. This system can be widely used in boiler, turbine and combined cycle, nuclear power and other medium of thermodynamic system and the simulation of fluid network, the mechanism of the type of module algorithm it established takes a good account of the fluid systems of quality, energy and momentum conservation relations[2]. The fluid network modeling techniques the software used have been used in hundreds of simulators.

\section{MUSE System Introduction}

MUSE is the abbreviations of English Multi-User Simulation Environment. MUSE system is a collection of software modules, for the large strong coupling and granular computing. Typical applications are power plant simulation system. Due to MUSE adopted Microsoft. Net framework design, therefore, compared with the traditional simulation support system, it is easier to link the DCS and the real-time control system of other power plant, and use simulation data provided by simulator to analyze and study. the control system of power plant.

MUSE system includes real-time database service (MUSE SDBSrv), real-time calculation engine (MUSE RTEngine), computer aided modeling system, the establishment of the terminal operator and run four parts. Computer aided modeling system also include five solutions: automatic thermal system modeling, control circuit configuration, automatic electric network modeling, ladder diagram modeling and general way of graphic configuration. MUSE also provide virtual DPU packages at the same time, the virtual DPU simulation package includes the ability to communicate with the actual DPU. 
MUSE in fully inherited the essence of the original system, such as high fidelity, full working condition of equipment model, fast, stable fluid network and electric network algorithms, based on the characteristics such as real-time and high-performance database, the extensive use of DCOM, Office plug technology, to satisfy the need of the power plant simulation training, control system research and so on, the system established convenient, low operation and maintenance costs.



Fig.1. The form of MUSE

\section{The Graphical Modeling Scheme of the Thermal System Solutions}

Thermal power system is always known as composed of a minority of several typical kinds of components(also known as the module). Therefore, the development of the graphical modeling thermal system software mainly includes the following content:

- Establish an equipment algorithm module library.

- Establish a resource library.

- Establish a management, configuration and its generating system.

A、 The Construction of the Module Library

For complex large system simulation modeling work, first to have done on the large system is according to its structure and function decomposition, decompose it into several classes of basic equipment or relatively complete description of a functional of the process unit, which is the basic module of the thermal system to be established in this paper. In other words, the division of the thermal system module equipment should have clear physical meaning, easy to identify and distinguish. A module represents a physical device, or on behalf of a physical device or part of a equipment group having certain independence and universality.

1)Module type: The module library established by this system according to the power plant equipment division method, the module according to the professional classification, mainly divided into the boiler, turbine, hydraulic turbine and general module. The module includes:

- About 60 boiler system module, such as evaporation system module, drum module, combustion module, superheater module, air preheater module, etc.

- About 60 turbine module, such as turbine module, heater module, deaerator module, vacuum module, turbine ontology modules, etc.

- More than 20 turbine module, such as tubular turbine, Francis turbine, surge shaft, reservoir and other modules.

- About 80 general module of fluid network, such as pipeline, valve, pump, fan and all kinds media of content source and sink points, etc.

2)Module Interface: Modules should be consistent with the boundary and interface for easy 
connection between each other. For most equipment modules, the module interface can completely be the nature interface of the working fluid flow between physical device or component part (s), for part of the module, in addition to working fluid interface, also consider the interface of information, such as the electrical interface of rotary machinery. The module interface point types this system designed shown in the table below, for any module in addition to the interface point, the corresponding algorithm point (FVA) is used to record constant and internal variables[3].

TABLEI: Style of connection of module

\begin{tabular}{|c|c|c|}
\hline Serial number & Types & Instructions \\
\hline 1 & FPA & For air medium \\
\hline 2 & FPC & For coal medium \\
\hline 3 & FPO & For oil medium \\
\hline 4 & FPS & For steam medium \\
\hline 5 & FPW & For water medium \\
\hline 6 & EC & Electrical interface for connecting device \\
\hline
\end{tabular}

B、The Algorithm of the Module:

For each module, when design the algorithm, we must follow the principle of mass conservation, momentum conservation and energy conservation, on the basis of their physical characteristics and the law of actual operation to design its algorithm. Including the general equation.

Energy conservation equation:

$\rho c V \frac{d T}{d \tau}=q_{\text {in }}+q_{\text {gen }}-q_{\text {out }}$

Mass conservation equation:

$V \frac{d \rho}{d \tau}=D_{\text {in }}+D_{\text {gen }}-D_{\text {out }}$

Momentum conservation equation:

$\frac{1}{\rho C_{v}^{2}}=p_{\text {in }}-p_{\text {out }}+f(w)$

Working medium state equation:

$f(p, v, t)=0$

Symbol description:

TABLE II: Symbol Description

\begin{tabular}{|c|c|c|c|}
\hline Symbol & Symbol Description & Symbol & Symbol Description \\
\hline$\rho$ & Media density $\mathrm{kg} / \mathrm{m}^{3}$ & $D_{\text {in }}$ & Inlet medium flow $\mathrm{kg} / \mathrm{s}$ \\
\hline$c$ & Media heat capacity $\mathrm{kj} / \mathrm{kg} \cdot \mathrm{c}$ & $D_{\text {out }}$ & Outlet medium flow $\mathrm{kg} / \mathrm{s}$ \\
\hline$V$ & Equipment volume $\mathrm{m}^{3}$ & $D_{\text {gen }}$ & $\begin{array}{c}\text { Medium flow generated by the } \\
\text { chemical reaction } \mathrm{kg} / \mathrm{s}\end{array}$ \\
\hline$T$ & Medium temperature ${ }^{\circ} \mathrm{C}$ & $p_{\text {in }}$ & Medium inlet pressure $\mathrm{MPa}$ \\
\hline$q_{\text {in }}$ & Input thermal flow $k j$ & $p_{\text {out }}$ & Medium outlet pressure $\mathrm{MPa}$ \\
\hline$q_{\text {out }}$ & Output thermal flow $k j$ & $f(w)$ & Power source $\mathrm{MPa}$ \\
\hline$q_{\text {gen }}$ & Produced thermal flow $k j$ & $C_{V}$ & $\begin{array}{c}\text { Surge absorption capability of the } \\
\text { equipment }\end{array}$ \\
\hline
\end{tabular}

C、 The Establishment of the Resource Database

The resource database this system had built up, is to support the calculation of module algorithm, such as water and steam thermodynamic properties function library, gas Enthalpy-temperature table, 
and a variety of interpolation algorithm and so on, these functions should have enough accuracy, the change of the parameter range is large enough, and not only to seek physical parameters such as enthalpy, density and entropy, also can calculate inverse function and partial derivative, etc. The water and steam function library system established conforms IMC97 standard, pressure range is 0 to $40 \mathrm{mpa}$, temperature range is $0 \sim 800{ }^{\circ} \mathrm{C}$. The temperature range of gas enthalpy temperature table is $0 \sim 2500{ }^{\circ} \mathrm{C}$, can meet the needs of the current power plant simulation.

D、The Solution of Fluid Network

Fluid network solution integrated in the MUSE platform, can realize automatic topology analysis of fluid network, distinguish node and branch, transfer module interface data, solve the correlation function of pressure and flow and so on, the provisions of this scheme (1) branch: fluid flows through a tunnel, pressure loss should between the inlet and outlet; (2) node: two and more than two branch intersection; (3) boundary point: a special kind of node, the pressure is the known conditions of fluid network equations to calculate .Flow net boundary point be selected should be coupling points of the pressure and flow in thermal system, such as the large volume point that pressure changes relatively slow, vapor liquid two phase coexistence point, etc. (4) source point: a special kind of node, only material outflow, and no material inflow, and the parameters of the outflow material remain unchanged, (5) the stuck points: a special kind of node, only material inflow, without material outflow. The general equation node and branch followed is as follows[4].

Mass conservation:

$C_{i} \frac{d p_{i}}{d t}=\sum_{j=1}^{k} I N P_{i j} G_{m}$

Momentum conservation equation:

$$
I_{m} \frac{d G_{m}}{d t}=I N P_{i j}\left(p_{j}-p_{i}+I N P_{i j} H_{m}\right)-R_{m} G_{m}\left|G_{m}\right|
$$

Energy conservation equation:

$$
M_{i} \frac{d H_{i}}{d t}=\sum_{j=1}^{k} \operatorname{Max}\left(I N P_{i j} G, 0\right) H_{j}-H_{i} \sum_{j=1}^{k} \operatorname{Max}\left(I N P_{i j} G_{m}, 0\right)+S_{i}
$$

Among them: $i$ and $j$ denote the node number, $m$ represents branch number.

Calculating fluid network using the modified gauss-seidel iterative method.

\begin{tabular}{|c|c|c|c|}
\hline Symbol & Symbol Description & Symbol & Symbol Description \\
\hline$p_{i}$ & Node pressure & $R_{m}$ & the resistance of the branch \\
\hline$H_{i}$ & the enthalpy of node & $I_{m}$ & mass inertial \\
\hline$C_{i}$ & compression factor & $I N P_{i j}$ & represent fluid network topology \\
\hline$M_{i}$ & quality of node & $I N P_{i j}=0$ & $\begin{array}{l}\text { represent there was no branch } \\
\text { between nodes } \mathrm{i} \text { and } \mathrm{j}\end{array}$ \\
\hline$S_{i}$ & $\begin{array}{l}\text { the node of the heat } \\
\text { dissipation }\end{array}$ & $I N P_{i j}=1$ & $\begin{array}{l}\text { represent there is a branch } \\
\text { between nodes } i \text { and } j \text {, its direction is } \\
\text { from } j \text { to } i\end{array}$ \\
\hline$G_{m}$ & Branch flow & $I N P_{i j}=-1$ & $\begin{array}{c}\text { represent there is a branch } \\
\text { between nodes } i \text { and } \mathrm{j} \text {, its direction is } \\
\text { from } \mathrm{i} \text { to } \mathrm{j}\end{array}$ \\
\hline$H_{m}$ & pump lift & & \\
\hline
\end{tabular}

TABLE III: Symbol Description

E、The Characteristics of Management Tools

1)High degree of openness

The module library, resource database and algorithm module interface of the MUSE graphical 
modeling system are completely open, the user can increase or decrease the module v the resource file and modify module algorithm, at the same time the module interface specification is defined for the physical objects, namely: if the user define a thermodynamic system equipment module, field definition of each interface point will be specified by the user, such as temperature, pressure, flow and other physical quantities. Further MUSE also provides the guide of write module .

2) Fully object-oriented model

As the auxiliary modeling system has built experience and knowledge of a large number of simulation calculation, modeling engineer just have corresponding professional knowledge to establish simulation model, the special field of module in numerical written in the module was given, or its scope is given, such as the influence coefficient of heating surface fouling, the coefficient of thermal conductivity of metal, the heat transfer coefficient of natural convection, etc. Professional engineers can directly use the existing module to lap the boiler and turbine simulation system, and don't need to care about the so-called computational problems, such as real time and stability of fluid network, also don't need to care about the topology of the flow net of lap joint system .

3) The convenient pretreatment function

In the actual modeling process, after each simulation system set up, and before run, the module we used need instantiated process, by instantiating the design data of simulation object and input the data used by the model algorithm into the database. MUSE provides a convenient pretreatment function, for each device and algorithm module, we can define its preprocessing algorithm in the module development phase, the result of pretreatment is automatically entered into the real-time database.

4) Powerful and convenient debugging function

In the debugging process of simulation model, it is necessary to tuning and testing the real-time data of simulation model, so MUSE provides powerful debugging function, you can online testing and modify any field of arbitrary module parameters through form and real time curve, can also use Visual Fortran to do source code level debugging of specific module, trace execution of each statement, can monitor all internal data at the same time.

5) Automatic document generation function

After modeling process is completed, MUSE can automatically generate maintenance documentation for each of the actual established simulation model, its format is HTML, you can easily according to directory inquiries, print and publish to the web page, document content including each system specification, simplifying assumptions, fault simulation, each illustrate of specific equipment, as well as the connection specification of the each module interface, using the function generated documentation, can basically meet the requirements of simulation model factory specifications.

6) Convenient search function

Automatic modeling compare with manual modeling, computer replaces the manual design code work, therefore, the interfaces between the system needs to be done with the aid of computer search, rather than rely on memory to complete, this system can complete search any system equipment and all kinds of interface, and can automatically generate the disk I/O interface table.

\section{Application Example}

On the MUSE graphical modeling platform established condensation turbine simulation model water system..First, according to the system flow chart, select the corresponding mold from mold library to set up simulation system, then for each mold instantiated, namely carry on the work of pretreatment the modules, calculate the data of module algorithm in the corresponding field, and then compile the mode, load and run the model, then you can debug the system, either through online monitoring parameters change of table or graph, also can be used to reproduce the parameters and modify, also can use software tools to debug the module source code[5]. With the development of the system simulation software as an example, using the graphical modeling tool 
than a modular modeling save time by $70 \%$.



Fig.2. Graphic Modeling Environment

\section{Conclusion}

Thermodynamic system graphical modeling system based on MUSE is based on fluid network algorithm and the modular modeling resources, develops configuration software such as module library, resource database and management tools that adapted graphical modeling, use the complete graphical auxiliary modeling software constructed by powerful graphics configuration function the Microsoft Visio software provided, provides about 160 thermal equipment modules, covering the power plant boiler, turbine and other host and auxiliary equipment in the system, fully able to meet the needs of the power station simulation, at the same time, the system has high degree of openness, according to the special needs of the practical work, can easily add new algorithm mold. The system has fully object-oriented model, convenient instantiation method, powerful debugging, document automatically generated and query function, it will be fully used in the work of simulator development in future.

\section{References}

[1] Lv Chongde, Ren Tingjin, Jiang Xuezhi,etc. "Simulation and modeling of large thermal power unit system"[M]. Tsinghua university press, 2002

[2] Ni Weidou, Xu Xiangdong, Li Zheng, etc. "Thermal power system modeling and control problems, science press", 1996.

[3] “DCOSE user manual, Gse-Unis(Beijing) Simulation Technology Co.,Ltd ., 2011.

[4] "MUSE user manual, UNI group(Beijing) intelligenct control science \& technology co.", LTD., 2013.

[5] "MUSE user manual, UNI group(Beijing) intelligenct control science \& technology co.", LTD., 2014 